\title{
Impact of DRX parameters on user equipment power saving and delay reduction in LTE networks
}

\author{
P. Arunagiri ${ }^{1 *}$, G. Nagarajan ${ }^{2}$ \\ ${ }^{1}$ Electronics and communication Engineering, SCSVMV University, Kanchipuram, India \\ ${ }^{2}$ Electronics and communication Engineering, Pondicherry Engineering College, Pondicherry, India
}

Corresponding Author Email: p.arunagiri@smvec.ac.in

https://doi.org/10.18280/ama_d.230101

Received: 29 October 2017

Accepted: 4 February 2018

\section{Keywords:}

DRX, DRX cycle, LTE, latency, power saving factor

\begin{abstract}
The growing need for the access of user equipment's (UE) has increased the necessity to investigate the power consumption in the switching process and to develop a method to reduce the power loss incurred in the system. Discontinuous reception mechanism (DRX) is a methodology proposed in Long Term Evolution (LTE) networks to achieve this desired effect. Although DRX mechanism introduces latency in the system, the power that can be saved in active and background traffic is comparatively good. This work focuses on power saving in UE and delay introduced in the process. Moreover, scheduling the DRX parameters can result in optimization of delay reduction and power. Thereby, better quality of service and enhanced lifetime of the UE can be achieved.
\end{abstract}

\section{INTRODUCTION}

Now-a-days, the use of mobile phones has increased extensively. The development of smart phones has grabbed the attention of the people as data transmission is made easy by enhancing the various utilities offered by the phone. Such advancements increased the need to transmit the data at a faster pace. The increasing demand for high speed data transmission network [1-5] has paved way for the proposal of Long Term Evolution by 3GPP.LTE mainly focusses on increasing the capacity and speed of the wireless data networks [6-9]. The LTE standard supports are high downlink rate of $300 \mathrm{Mbit} / \mathrm{s}$ and uplink rate of $74.6 \mathrm{Mbit} / \mathrm{s}$. LTE networks also provides more throughput than High Speed Packet Access [10$13]$.

There is a higher consumption of power by the user equipment to achieve higher data rates using LTE. This results in frequent charging and discharging of the battery which eventually reduces the battery life. To solve this problem and to reduce the power consumption, a mechanism called Discontinuous Reception (DRX) [14] is used. Usually, the UE is turned $\mathrm{ON}$ all the time to monitor the network to decode physical downlink control channel (PDCCH).

In DRX mechanism when there is no traffic, monitoring is not required and the UE can enter into power saving mode. Though DRX mechanism saves power [15], it also induces delay into the system. The delay is tolerable in case of background data traffic but for active data traffic, this can result in loss of data.

\section{DRX MECHANISM}

The two possible states for LTE devices are idle state and connected state. When there is data activity either uplink or downlink, the device stays in connected state. At the end of data transmission, the device moves to idle state after the expiry of a timer called RRC Inactivity timer. The timer is reset every time there is a data exchange. There is no transmission or reception of data during idle state. An UE in idle performs selection and reselection of geographic site. Paging is a procedure to wake up the UE for data activity and is triggered by eNB in Radio Access Network which establishes a connection between the UE and the base station.

The user equipment monitors the Physical Downlink Control Channel for any data activity. Discontinuous reception is a mechanism to reduce the power loss incurred in the system and at the same time extend the battery life. DRX mechanism also uses a timer to drive the UE into the sleep period. The sleep period is classified into light sleep and deep sleep to employ effective power saving.

\subsection{Light sleep}

The LTE device uses a timer called DRX short cycle timer to wake up the device after short sleep to check the PDCCH for any packet activity. If the device detects any downlink data during the $\mathrm{ON}$ time, the device moves to connected state and the exchange of data is initiated. In other case, the device sleeps until the next ON time.

\subsection{Deep sleep}

Similarly, the LTE device uses a timer named DRX long cycle timer to check PDCCH for packet activity after long sleep. The Timer starts running after device has been in many cycles of light sleep. The device sleeps if there is no packet activity until the next $\mathrm{ON}$ time.

\section{SEMI-MARKOV PROCESS AND MODELING}

The arrival of the data packets [16] is considered to follow semi Markov method and other parameters are opted as indicated in Table 1. The UE stay's in any of the state's S1, S2 or S3: 


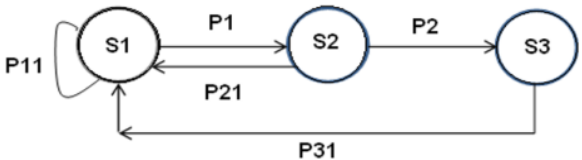

Figure 1. State transition diagram

$\mathrm{S} 1$ is the active state in which the UE continuously receives data packets and the UE consumes power during data activity.

$\mathrm{S} 2$ is light sleep state in which the UE is in short DRX cycle. When there is no packet activity for time tinact after the after the reception of data during the state $\mathrm{S} 1$.

$\mathrm{S} 3$ is deep sleep state in which the UE is in long DRX cycle. The UE enters state S3 after several occurrences of short DRX cycles [17-19] and the UE is said to be in power saving mode.

Table 1. Parameters and distribution model utilized

\begin{tabular}{ccc}
\hline Parameter & Distribution Model & Mean value \\
\hline $\begin{array}{c}\text { Inter-session idle time } i s \\
\mathrm{t}_{\mathrm{is}}\end{array}$ & Exponential & $1 / \mathrm{L}_{\mathrm{is}}$ \\
$\begin{array}{c}\text { Number of packet calls } \\
\text { per session } \mathrm{N}_{\mathrm{pc}}\end{array}$ & Geometric & $\mathrm{U}_{\mathrm{pc}}$ \\
$\begin{array}{c}\text { Inter-packet call idle time } \\
\mathrm{t}_{\mathrm{ipc}}\end{array}$ & Exponential & $1 / \mathrm{L}_{\mathrm{ipc}}$ \\
$\begin{array}{c}\text { Number of packets per } \\
\text { packet call } \mathrm{N}_{\mathrm{p}}\end{array}$ & Geometric & $\mathrm{U}_{\mathrm{p}}$ \\
$\begin{array}{c}\text { Inter-packet arrival time } \\
\text { tip }\end{array}$ & Exponential & $1 / \mathrm{L}_{\mathrm{ip}}$ \\
\hline
\end{tabular}

Let $\mathrm{U}_{\mathrm{pc}}$ be the number of packets arriving per session, then the probability that next packet belongs to the current session (case 1) is given by

$P_{p c}=1-1 / U_{p c}$

The probability that the next packet is the initial packet of the subsequent session (case 2) is given by

$P_{s}=1 / U_{p c}$

The probabilities are based on the memoryless property of the geometric distribution. The state transition probabilities are given below:

The probability that the packet arrives before the inactivity period $\left(t_{i}\right)$ expires for case 1 is given by

$q_{1}=1-e^{(-L i p c * t i)}$

For case 2, it is given by

$q_{2}=1-e^{(-L i s * t i)}$

The Transition probability from state to state could be deduced with following considerations
In state $\mathrm{S}_{1}, \mathrm{~T}_{\mathrm{I}}$ (DRX Inactivity Timer) will run

To Stay in state 1 , ts and tpc should be lesser than $T_{I}$

The conditions for state $S_{1}$ are:

Ts $<\mathrm{T}_{\mathrm{I}}$ in new session with probabilities $\mathrm{P}_{\mathrm{ns}}$

Tps $<\mathrm{T}_{\mathrm{I}}$ in ongoing session with probabilities $\mathrm{p}_{\mathrm{os}}$

The parameters involved in state transition are assigned as mentioned in Table 2

Table 2. Parameters and assigned names

\begin{tabular}{cc}
\hline Parameters & Assigned Names \\
\hline $\mathrm{P}_{\mathrm{r}}(\mathrm{r}$ & Probability of occurrence \\
$\mathrm{T}_{\mathrm{pc}}$ & Packet call inter arrival time \\
$\mathrm{T}_{\mathrm{I}}$ & DRX Inactivity timer \\
$\mathrm{P}_{\mathrm{os}}$ & Ongoing Session Probability \\
$\mathrm{T}_{\mathrm{s}}$ & Session inter Arrival time \\
$\mathrm{P}_{\mathrm{ns}}$ & next session probability \\
\hline
\end{tabular}

$\mathrm{P}_{11}$ : Transition probability in retrieving the state (Generally Transition probability from state X to State $\mathrm{Y}$ ).

Therefore, the probability that the UE in state $\mathrm{S} 1$ continues to be in state $\mathrm{S} 1$ is given by

$P_{11}=P_{p c} * q_{1}+P_{s} * q_{2}$

The probability that the UE in state $\mathrm{S} 1$ enters into state $\mathrm{S} 2$ after inactivity timer has expired is given by

$P_{12}=P_{p c} *\left(1-q_{1}\right)+P_{s} *\left(1-q_{2}\right)$

When the DRX inactivity timer expires, the UE enters into state S2 and the DRX short cycle timer is activated. If there is a packet activity before the expiry of the DRX short cycle timer, the timer is cancelled and the UE is pushed back to active state $\mathrm{S} 1$. The probability that the packet arrives before the expiry of DRX short cycle timer $\left(t_{n}\right)$ [20] is given by,

$q_{3}=1-e^{(-L i p c * t n)},($ for case 1$)$

$q_{4}=1-e^{(-L i s * t n)},($ for case 2$)$

The probability that the UE in state $\mathrm{S} 2$ moves to state $\mathrm{S} 1$ is given by,

$P_{21}=P_{p c} * q_{3}+P_{s} * q_{4}$

When the UE in state S2 expires no packet activity for one or several short DRX cycle based on the formulation of parameters, the UE moves into Long DRX cycle i,e., state S3 [17]. The probability that the UE in state S2 moves to state S3 is given by

$P_{23}=P_{p c} *\left(1-q_{3}\right)+P_{s} *\left(1-q_{4}\right)$

The state transition probability matrix is expressed as $\mathrm{P}$

$$
P=\left[\begin{array}{ccc}
P_{p c} * q_{1}+P_{s} * q_{2} & P_{p c} *\left(1-q_{1}\right)+P_{s} *\left(1-q_{2}\right) & 0 \\
P_{p c} * q_{3}+P_{s} * q_{4} & 0 & P_{p c} *\left(1-q_{3}\right)+P_{s} *\left(1-q_{4}\right) \\
0 & 0 & 1
\end{array}\right]
$$

If $\mathrm{P}_{\mathrm{i}}$ represents the probability that the UE is in the state $\mathrm{S}_{\mathrm{i}}$, then the

$\sum_{i=1}^{3} P_{i}=1$
Let $\mathrm{H}_{\mathrm{i}}$ the holding time in the state $\mathrm{S}_{\mathrm{i}}$. The holding time in state $\mathrm{S} 2$ is given by 
$E_{H 2}=\left(P_{p c} e^{\left(-L_{\left.i p c^{*} t_{n}\right)}\right.}+P_{s} e^{\left(-L_{i s} * t_{i}\right)}\right) N t_{d s}+\left(P_{p c} e^{\left(-L_{\left.i p c^{*} t_{n}\right)}\right.}+P_{s} e^{\left(-L_{i s} * t_{n}\right)}\right)\left(\frac{P_{p c}}{1-e^{\left(-L_{i p c^{* t} d s}\right)}}+\frac{P_{s}}{\left.1-e^{\left(-L_{i s} * t\right.} d s\right)}\right) t_{d s}$

The holding time in state $\mathrm{S} 2$ is given by

$E_{H 3}=\left(\frac{P_{p c}}{1-e^{\left(-L_{i p c^{*} t}\right)}}+\frac{P_{S}}{1-e^{\left(-L_{i s^{*}} d l\right)}}\right) t_{d l}$

$\left.E_{H 2}^{\prime}=\left(P_{p c} e^{\left(-L_{\left.i p c^{*} t_{n}\right)}\right.}+P_{s} e^{\left(-L_{i s} * t_{i}\right)}\right) N\left(t_{d s}-t\right)+\left(P_{p c} e^{\left(-L_{\left.i p c^{*} t_{n}\right)}\right.}+P_{s} e^{\left(-L_{i s} * t_{n}\right)}\right)\left(\frac{P_{p c}}{1-e^{\left(-L_{i p c^{* t} d s}\right)}}+\frac{P_{s}}{\left.1-e^{\left(-L_{i s} * t\right.} d s\right)}\right)\left(t_{d s}-t\right)_{\mathrm{ds}}-\mathrm{t}\right)$

$E_{H 3}^{\prime}=\left(\frac{P_{p c}}{1-e^{\left(-L_{i p c^{* t}} d l\right)}}+\frac{P_{S}}{\left.1-e^{\left(-L_{i s} * t\right.} d l\right)}\right)\left(t_{d l}-t\right)$

The power saving factor $\mathrm{P}$ [7] is defined by the ratio of the effective sleep time of UE in short and long DRX cycle to the holding time of UE in short and long DRX cycle [21-22].
Let $t$ be the ON duration for which the UE checks for packet activity during the DRX cycle. So the effective sleeping time during the short and long DRX cycles is given as $t_{d s}-t$ and $t_{d l}-t$. So the effective sleep time of the UE in short and long DRX cycles are given by,

$p_{i}=\left\{\begin{array}{c}P_{p c} e^{-\lambda_{i p c} t_{l}} e^{-\lambda_{i p c}(i-1) t_{D S}}\left(1-e^{-\lambda_{i p c}(i-1) t_{D S}}\right)+P_{S} e^{-\lambda_{i s} t_{s}} e^{-\lambda_{i s}(i-1) t_{D S}}\left(1-e^{-\lambda_{i s} t_{D S}}\right), i \leq N . \\ P_{p c} e^{-\lambda_{i p c} t_{l}} e^{-\lambda_{i p c} N t_{D S}} e^{-\lambda_{i p c}(i-N-1) t_{D L}}\left(1-e^{-\lambda_{i p c}(i-1) t_{D S}}\right)+P_{S} e^{-\lambda_{i s} t_{s}} e^{-\lambda_{i s}(i-1) t_{D S}}\left(1-e^{-\lambda_{i s} t_{D S}}\right), i>N .\end{array}\right.$

As the inter packet idle time and the inter packet session time is exponentially distributed, the packet call arrivals are supposed to be Poisson distribution [23-25].

The mean wake up delay is derived as

$D=\sum_{i=1}^{N}\left(p_{i} t_{d s} / 2\right)+\sum_{i=N+1}^{\infty}\left(p_{i} t_{d l} / 2\right)$

The parameters involved in the mathematical models could be iterated for different traffic models based on offered services or required QoS.

\section{SIMULATION RESULTS}

The various parameters used for simulation are listed in Table 3.

Table 3. Parameters considered for simulation

\begin{tabular}{ccc}
\hline SL:NO & PARAMETER & DETAIL \\
\hline 1 & Tool & MATLAB R2015 a \\
2 & $\mathrm{~L}_{\mathrm{ip}}$ & Inter packet arrival time \\
3 & $\mathrm{~L}_{\mathrm{ipc}}$ & Inter-packet call idle time \\
4 & $\mathrm{~L}_{\mathrm{is}}$ & Inter-session idle time \\
5 & $\mathrm{U}_{\mathrm{p}}$ & Number of packets per packet call \\
6 & $\mathrm{U}_{\mathrm{pc}}$ & Number of packet calls per session \\
7 & $\mathrm{~T}_{\mathrm{dl}}$ & DRX long cycle \\
8 & $\mathrm{~T}_{\mathrm{I}}$ & DRX Inactivity timer \\
9 & $\mathrm{~T}_{\mathrm{ds}}$ & DRX short cycle \\
10 & $\mathrm{~N}$ & No. of short DRX cycles \\
\hline
\end{tabular}

The simulation parameters and their values used for simulation is listed in Table 3. The variation of power saved with respect to long and short DRX cycles are shown in the Figure 2. The power saving versus DRX long cycle is a concave down increasing graph. Power saving increases with increasing values of long DRX cycle. This is because the ON period for which the UE monitors the Physical downlink control channel-(PPDH) is constant and the time spent by the user equipment in sleep mode is increased as DRX long cycle increases, thus saving more apparent power when compared to active and DRX short cycle.
Table 4. Parameters considered for simulation

\begin{tabular}{ccc}
\hline SL:NO & PARAMETER & VALUES \\
\hline 1 & $\mathrm{~L}_{\mathrm{ip}}$ & 10 \\
2 & $\mathrm{~L}_{\mathrm{ipc}}$ & $1 / 30$ \\
3 & $\mathrm{~L}_{\mathrm{is}}$ & $1 / 2000$ \\
4 & $\mathrm{U}_{\mathrm{p}}$ & 25 \\
5 & $\mathrm{U}_{\mathrm{pc}}$ & 5 \\
6 & $\mathrm{~T}_{\mathrm{dl}}$ & 20 \\
7 & $\mathrm{~N}$ & 1 \\
\hline
\end{tabular}

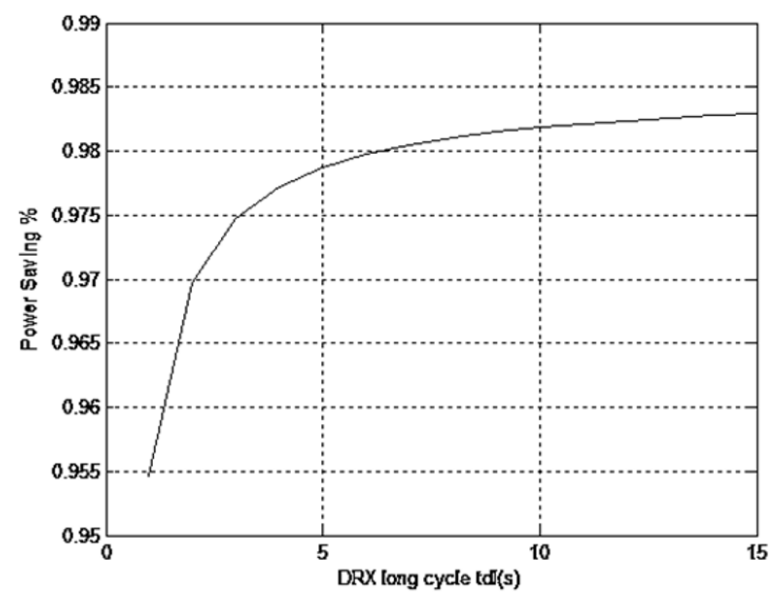

Figure 2. $\operatorname{Lip}=10, \mathrm{Lipc}=1 / 30, \mathrm{Lis}=1 / 2000, \mathrm{Upc}=5, \mathrm{Up}=25$, $\mathrm{tn}=40, \mathrm{tdl}=20, \mathrm{~N}=1, \mathrm{t}=.1, \mathrm{t} 1=2$

Figure 3 shows the variation of mean wake up delay with respect to Long DRX cycle. It shows that the delay incurred in the system due to the DRX mechanism increases proportionally with DRX long cycle. Delay is introduced in the system by the worst case probability that the call arrives during the Long DRX sleep. As the sleep time increases the time required to establish a call also increases proportionally. This is because the call is detected only in the next ON period of long DRX cycle. 


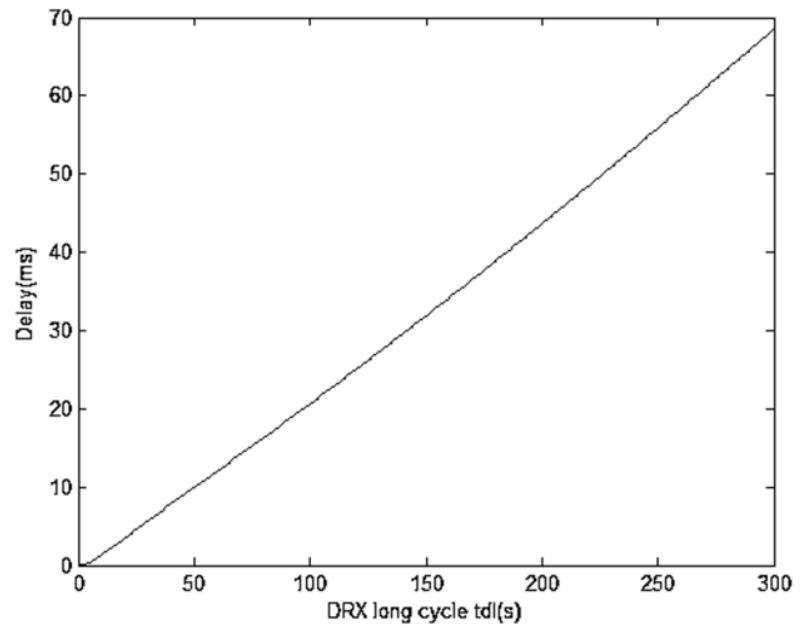

Figure 3. $\mathrm{Lip}=10, \mathrm{Lipc}=1 / 30, \mathrm{Lis}=1 / 2000, \mathrm{Upc}=5, \mathrm{Up}=25$, $\mathrm{tn}=10, \mathrm{tdl}=2, \mathrm{~N}=1, \mathrm{t}=.1, \mathrm{t} 1=2$

Figure 4 shows the variation of power saving with DRX short cycle. The comparison of power saving versus DRX short and long cycle shows that, UE spends more time in the sleep mode in DRX long cycle than in DRX short cycle. Therefore, the power that be saved in an UE is more in DRX long cycle compared to DRX short cycle.

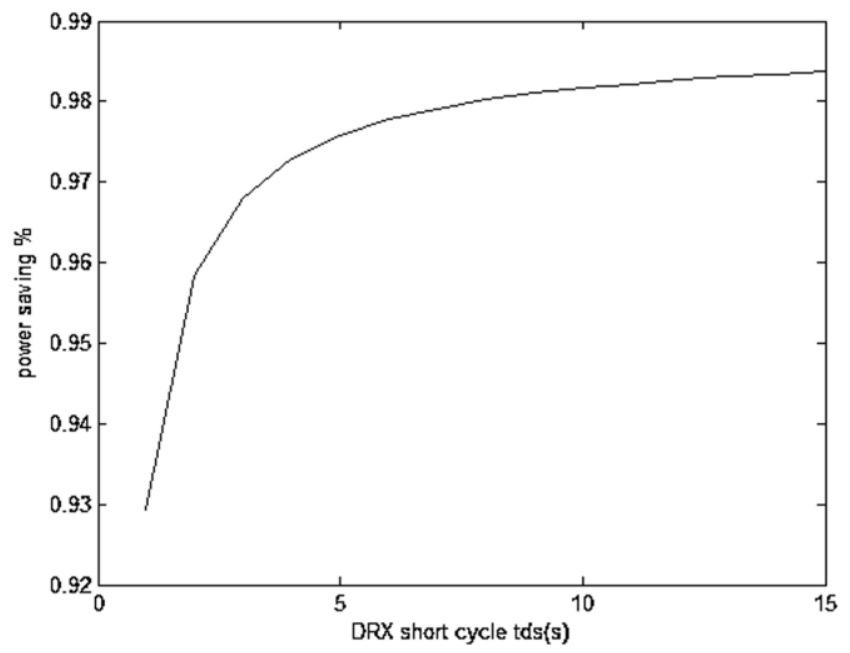

Figure 4. $\mathrm{Lip}=10, \mathrm{Lipc}=1 / 30, \mathrm{Lis}=1 / 2000, \mathrm{Upc}=5, \mathrm{Up}=25$, $\mathrm{tn}=10, \mathrm{t} 1=2, \mathrm{tds}=2, \mathrm{~N}=4, \mathrm{t}=.04$

Similar to that of Long DRX cycle, the power saving increases as DRX short cycle increases and the ON duration for which the UE checks for PDCCH is kept constant.

Figure 5 shows the variation of mean wake up delay with respect to DRX short cycle. It shows that delay introduced into the system during a DRX short cycle is less compared to that in DRX long cycle. As the DRX short cycle i.e the sleep time increases, the delay also increases. The duration for which the UE monitors the PDCCH is constant and the delay increases as the sleep time increases. This is because the call arrival is known only during the next $\mathrm{ON}$ duration. Therefore, if the sleep time increases the delay also increases.

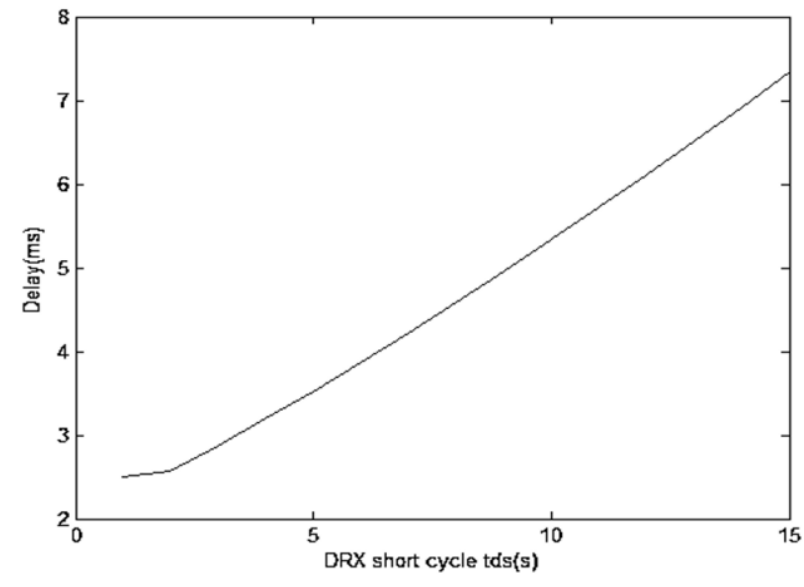

Figure 5. $\mathrm{Lip}=10, \mathrm{Lipc}=1 / 30, \mathrm{Lis}=1 / 2000, \mathrm{Upc}=5, \mathrm{Up}=25$, $\mathrm{tn}=120, \mathrm{tdl}=20, \mathrm{~N}=104, \mathrm{t}=1, \mathrm{t} 1=2$

\section{CONCLUSION}

The DRX mechanism in LTE drives the UE into sleep mode while there is no packet activity for a prolonged period. It is validated to save the battery power to the maximum probable extent. There is a reduction in the charging and discharging of battery as well, hence the battery life is also extended. The simulation results for power saving $\% \mathrm{Vz}$ DRX short cycle and Long cycles validate the power saving percentage is up to $98 \%$, hence the UE could be used 4 fold times longer than the estimated time of usage. Proper modelling of data arrival rate for different services will also yield similar power saving $\%$. But the mechanism also induces a delay into the system which is also studied in the paper. The data services which have a tolerable delay from $7.4 \mathrm{~ms}$ to $68 \mathrm{~ms}$ can be accommodated with LTE -DRX mechanism rather than fully connected system. In order to compromise between the two obtained characteristics of the system, a relationship is developed between power saving factors and the delay incurred in the system.

\section{REFERENCES}

[1] Zayyat K, Ababneh I, al-Dubai A. (2000). A new routing approach for collective communication in interconnection networks. Advances in Modelling \& Analysis D 5(4). https://doiorg/10.3168/jds.S00220302(03)73636-4

[2] Aqel MJ. (2001). Optimal routing expert system for computer networks. Advances in Modelling \& Analysis B 44(3-4): 59-67.

[3] Ababneh I. (2002). Multicast algorithms for All-Port wormhole-routed 3D mesh networks. Advances in Modelling and Analysis D 7(3): 1-18.

[4] Qureshi MF, Chhalotra GP, Mahajan RS. (2003). A study of reliability attributes of neural controlling networks using fuzzy logic error detection during training. AMSE Journal, Advances B 46(4): 19-40.

[5] Aqel MJ. Hatem MS. (2001). An expert system for computer network design based on MENTOR algorithm. Journal of Modelling - Measurement \& Control 22(1-2): 11-20.

[6] Adham NZ, El-Hady AA, Naim N. (2004). A new approach of evaluation network performance based on 
routing algorithms estimation. Advances in Modelling and Analysis D 9(1): 41-54.

[7] Nsayef SA, Mahmoud WA. (2004). Performance analysis of a proposed computer network. Advances in Modelling and Analysis D 9(2): 41-53.

[8] Peer S.K. Sharma DK, Rivindranath K, Naidu MM. (2004). Message routing problem with traffic based scheduling in networks. Advances in Modelling and Analysis D 9(3): 1-14.

[9] Beghdad R. (2004). A minimum-cost maximum-flows QoS routing protocol for multicast communication networks. Advances in Modelling and Analysis D 9(4): 41-52.

[10] Beghdad R. (2005). Performances analysis of a LLC protocol of a home building network with mobile stations. Advances in Modelling and Analysis D 10(2): 19-30.

[11] Pujeri RV, Sivanandam SN. (2005). Design and performance evaluation of ATM congestion control using neural networks. Advances in Modelling and Analysis D 10(3): 27-56.

[12] Lewoc JB, Izworski A, Skowronski S. (2005). An approximate actual network performance evaluation method. Advances in Modelling and Analysis D 10(4): 1-12. https://doi.org/10.1109/ISMS.2010.74

[13] Rahaman H, Das DK. (2005). Modelling ESOP networks for detecting multiple stock - at faults. Advances in Modelling and Analysis D 10(4): 25-36.

[14] Wang K, Li X, Ji H. (2016). Modeling and optimizing the LTE discontinuou reception mechanism under selfsimilar traffic. IEEE Transactions on Vehicular Technology 65(7): 5595-5610.

[15] Koc AT. (2014). Device power saving and latency optimization in LTE-A networks through DRX Configuration. IEEE Transactions on Wireless Communications 13(5): 2614-2625. https://doiorg/10.1109/TWC.2014.031914.131298

[16] Yang SR, Yan SY, Hung HN. (2007). Modeling UMTS power saving with bursty packet data traffic. IEEE
Transaction, Mobile Comp. 6(12): 1398-1409. https://doiorg/10.1109/tmc.2007.1072

[17] Yangnd SR, Lin YB. (2005). Modeling of UMTS discontinuous reception mechanism. IEEE Transaction. Wireless Comm. 4(1): 312-319. https://doiorg/10.1109/TWC.2004.840259

[18] Abeta S. (2010). Radio access network development department NTT DOCOMO, INC. Toward LTE Commercial Launch and Future Plan for LTE Enhancements, 146-150.

[19] Sangkyu B, Choi BD. (2011). Analysis of discontinuous reception (DRX) with both downlink and uplink packet arrivals in 3GPP LTE. Proceedings of the 6th International Conference on Queueing Theory and Network Applications (4): 841-86. $10.1145 / 2021216.2021218$

[20] Zhou KJ, Nikaein N, Spyropoulos T. (2012). LTE/LTEa discontinuous reception modeling for machine type communications. IEEE Wireless Communications Letters $2(1)$ : 102-105. https://doi.org/10.1109/WCL.2012.120312.120615

[21] Aho K, Henttonen T, Puttonen J. (2011). User equipment energy efficiency verses LTE network performance. IJAT (3-4): 140-151.

[22] Aho K, Henttonen T, Puttonen J. (2010). Trade-off between increased talk-time and LTE performance. IEEE Computer Society, 371-375.

[23] Zhou L, Xu HB, Tian H, Gao YJ. (2008). Performance Analysis of power saving mechanism with adjustable DRX cycles in 3GPP LTE. 2008 IEEE 68th Vehicular Technology Conference. https://doiorg/10.1109/VETECF.2008.312

[24] Bontu CS, Nortel EI. (2009). DRX mechanism for power saving in LTE. IEEE Communications Magazine 47(6): 48-55. https://doiorg/10.1109/MCOM.2009.5116800

[25] Yu Y.P, Feng KT. (2012). Traffic-based DRX cycles adjustment scheme for 3GPP LTE systems. 2012 IEEE 75th Vehicular Technology Conference (VTC Spring). https://doiorg/10.1109/VETECS.2012.6240136 